\title{
A single case study of biofeedback training in an adolescent with cancer-related pain
}

\begin{abstract}
Background: Management of cancer pain associated with anxiety derived is complex, especial in adolescent age when the ability to have body control gains a really central role.

This case report aims to provide health professionals with insight into how patients' awareness of physiological processes - improved by means of an individualized Biofeedback-Assisted Relaxation Training (BART) protocol along 4 weeks of treatment - can modify patients' perception of pain. Results from this case report suggest the opportunity for further development of a multidimensional clinicalreasoning framework to treat cancer pain in pediatric involving also biofeedback treatment.
\end{abstract}

Keywords: biofeedback-assisted relaxation training, cancer pain, adolescent, case report
Volume 8 Issue 6 - 2018

\author{
Alessandro Failo,' Mariantonietta Mazzoldi, ${ }^{2}$ \\ Laura Battisti, ${ }^{3}$ Lydia Pescollderungg, ${ }^{3}$ Paola \\ Venuti' \\ 'Department of Psychology and Cognitive Sciences, University \\ of Trento, ITALY \\ ${ }^{2}$ Clinical Psychological Unit, Health District of Bolzano, Italy \\ ${ }^{3}$ Department of Pediatrics, Regional Hospital of Bolzano, Italy
}

Correspondence: Alessandro Failo, Department of Psychology and Cognitive Sciences, University of Trento,Via Matteo del Ben 5/B, 38068, Rovereto, ITALY, Tel +39 0464 808I03, Email a.foilo@unitn.it

Received: July 30, 20I8 | Published: November 20, 2018

\section{Introduction}

Daily challenges faced by adolescents diagnosed with cancer are many, and they become even more difficult when the patients are in pain. Both acute (from procedures) and chronic (from cancer itself or treatments) pain has been recognized as one of the most frequent and distressing symptoms in cancer which adversely affects the quality of life and the ability to control one's own body. ${ }^{1,2}$ Cancer related pain (in a broader sense) can elicit or is associated to a variety of somatic symptoms such breathlessness, muscle tension, dizziness, palpitation and anxiety. ${ }^{3}$ It is well known that decreased anxiety in pediatric age is not only associated with decreased distress and pain during invasive medical procedures, but also with less negative attitudes towards future medical procedures and pain episodes. ${ }^{4}$ It is evident that the main approach in the management of cancer pain in children is surely the pharmacological one, but it could be more efficacious in association with non-pharmacological techniques. ${ }^{5}$ Among the non-pharmacological approaches, those that include biofeedback (a tool that measures and feeds back information about an individual's physiological activity) can be very helpful in teaching children through better body control how to manage pain and anxiety, especially when these are related to invasive procedures. In fact, biofeedback affects resonance and oscillations of different feedback loops in the central nervous system ${ }^{6}$ and when it is delivered together with relaxation therapy or autogenic training seems to be effective in reducing the frequency of pain episodes in the pediatric age. ${ }^{7}$ Several BiofeedbackAssisted Relaxation Training programs (BART) have been applied in a wide range of pediatric conditions, including Headache, ${ }^{8}$ Irritable Bowel Syndrome and Functional Abdominal Pain, ${ }^{9}$ pain in Sickle Cell Disease, ${ }^{10}$ Burn Jnjury, ${ }^{11}$ and also for face with anxiety and stress-related medical conditions to learn to relax by the control of physiological reactivity. ${ }^{12}$ However, to date, little research exists about the use of Biofeedback-based treatments for children with cancer related pain. A good example in this field is the study conducted by Shockey, ${ }^{13}$ who found that 4 -session intervention combining relaxation and biofeedback in a clinical setting may be beneficial to children experiencing procedural distress as a novel coping strategy. Furthermore, over the last decade, new biofeedback tools have been developed with video-game-like technology interfaces. This allows to an immediate experience, in real-time, adapting different kinds of feedback to increase interest and engagement, thus facilitating the learning of deep breathing, heart rate variability (HRV) and muscle relaxation. ${ }^{12}$

From these considerations, the objective of this single case report is to illustrate how biofeedback can be integrated in an Inter professional Care Team, in a format that is acceptable within a specific treatment time frame.

\section{Methods}

\section{Procedures}

Since effective treatments is a collaborative effort among services, University of Trento in partnership with Bolzano's Hospital have developed the SMART4Pain program to manage chronic pain in adolescents which started in December 2017 by the Ethical approval obtained from the Hospital Committee. From it, we planned another feasibility study, which has been adapted to treat cancer- and treatments-related pain, within DH routine practices. Using a case example, we want show how biofeedback can be integrated as a part of a multidisciplinary care modality.

In addition to his standard medical care, the adolescent completed 4 sessions of Biofeedback treatment to increase awareness of and control over one's physiological processes. The patient was encouraged to perform the exercises learned in the session at home at least three times a week.

\section{Physiological data acquisition and parameters considered}

The biofeedback device that we have used is a ProComp5 Infiniti System w/Bio Graph Infiniti Software - T7525, which meets the requirements concerning medical devices as stated in the European Community Council Directive 93/42/EEC, which monitors and records autonomic nervous activity with 5 channels: surface electromyography (sEMG), blood volume pulse (BVP), skin conductance (SC), peripheral temperature (TEMP), and respiratory response (RESP). 
Our protocol for the assessment and training of self-control of physiological functions was based on a pre-post session-by-session evaluation combining relaxation training and practice (deep breathing and mini-progressive muscle relaxation) with psychoeducation about pain mechanisms and how to distress, who explained by hyperactivity of the Sympathetic Nervous System, may exacerbate pain and inflammation. Then, how different kinds of relaxation can relieve or prevent stress and when and how to use relaxation techniques in the hospital or when the patient is in pain.

For the purpose of this article we focus only on physiological parameters and not on other sources (such questionnaires, as well clinical interview - which are naturally still important).

The two major physiological parameters we have considered are:

i. Heart Rate Variability (HRV), which is the constant variation in Heart Rate (HR) measured in terms of the interbeat intervals
(IBIs). The SD of these IBIs is called SDNN when computed after all artifacts have been "corrected". ${ }^{14}$ The training provided was aimed to increase respiratory sinus arrhythmia (RSA) which directly influences HRV, given RSA is the fluctuation in heart rate corresponding to breathing, with heart rate increasing with inhalation and decreasing with exhalation. ${ }^{15,16}$ High levels of HRV reflect a better vagal activity and it is known that vagus nerve influences the modulation of pain. ${ }^{17}$

ii. sEMG amplitude and frequency parameters of muscular activity, because they are useful measures which can reflect specific stress tender point patterns. ${ }^{18}$ By sEMG biofeedback equipment the objective was to measure the muscle use during all 4 sessions of training. Furthermore, from the $3^{\circ}$ session, training had thus aimed to increase modulation of neck muscle activity for better control muscle aches, generally related to stress (anticipatory and residual) (Table 1).

Table 1 Biofeedback-assisted relaxation training intervention plan

\begin{tabular}{|c|c|c|}
\hline $\mathrm{N}^{\circ}$ of session & Activities & Assessment \\
\hline Session 1 (60minutes) & $\begin{array}{ll}\text { - } & \text { Education about Physiology of Pain } \\
\text { and Hyperactivity of the Sympathetic } \\
\text { Nervous System, } \\
\text { - } \quad \text { Mini Deep-breathing exercises } \\
\text { - } \quad \text { After Resonance frequency assessment, } \\
\text { Breathing with pacer } \\
\text { Tips for home practice through use of a } \\
\text { Breathing app } \\
\text { - } \quad \text { Pain diary for home } \\
\quad \text { Set Therapeutic goal }\end{array}$ & $\begin{array}{l}\text { - Initial Semi-structured interviews (pain } \\
\text { location, onset, qualities) } \\
\text { PedsQL } \\
\text { - } \quad \text { life) } \\
\text { - } \quad \text { VAD (anxiety and depression trait) } \\
\text { - } \quad \text { Baseline and Analogue Scale - pain) } \\
\text { sEMG, SC, Temp, Resp rate }\end{array}$ \\
\hline Session 2 (60minutes) & $\begin{array}{ll}\text { - } & \text { Review about use of Breathing app and } \\
& \text { pain diary } \\
\text { - } & \text { Specific explanations about HRV } \\
\text { - } & \text { Breathing with pacer and without } \\
& \text { Breathing with power (LF) }\end{array}$ & $\begin{array}{l}\text { - } \quad \text { VAS } \\
\text { Baseline and post: HR, SDNN, power, } \\
\text { sEMG, SC, Temp, Resp rate }\end{array}$ \\
\hline Session 3 (60minutes) & $\begin{array}{ll}\text { - } & \text { Review about use of Breathing app and } \\
\text { pain diary } \\
\text { - }\end{array}$ & $\begin{array}{l}\text { - } \quad \text { VAS } \\
\text { Baseline and post: HR, SDNN, power, } \\
\text { sEMG, SC, Temp, Resp rate }\end{array}$ \\
\hline Session 4 (60minutes) & $\begin{array}{ll}\text { - } & \text { Review about use of Breathing app and } \\
\text { pain diary } \\
\text { - } \\
\text { Breathing with pacer and without } \\
\text { Breathing with power } \\
\text { - } \quad \text { EMG Biofeedback training } \\
\text { Review specific doubts/difficults and } \\
\text { suggestion for prevent pain flare-ups } \\
\text { Review Therapeutic goal }\end{array}$ & $\begin{array}{ll}- & \text { PedsQL Cancer quality of life) } \\
\text { - } & \text { TAD (anxiety and depression trait) } \\
\text { - } & \text { BAS } \\
& \text { Baseline and post: HR, SDNN, power, } \\
\text { sEMG, Temp, Resp rate }\end{array}$ \\
\hline
\end{tabular}

\section{Results}

\section{Case study description}

'Mariano' is a male teenager with Acute Lymphocytic Leukemia (ALL). He is in the phase of re-induction chemo immunotherapy and his main symptoms are pain mainly localized to the head and of the muscles of the neck (a couple of time per week usually) and occasionally around hips and knees.

He also presented weakness, general numbness and usually he referred to have a significant anxiety during medical procedures. 
He followed the treatment protocol planned, with the agreed target to increase his physiologic self-regulation to face in a better way his pain-related anxiety during invasive procedures and treatments.

At the first session he was taught abdominal (diaphragmatic) breathing exercises to use less chest respiration and unblock the diaphragm. Then we looked for his resonance frequency (heart rate and breathing synchronization) identified at a rate of $7.5 \mathrm{breaths} / \mathrm{min}$, and afterwards he was trained with a breathing pacing devices (with visual and auditory feedback).

He continued practicing at home with a breathing app (a guide for inhale and exhale) with his exactly breathing rate applied during the session, for at least once a day. This he performed for the entire 4 weeks of treatment.

Session two was aimed to increase RSA with and without breath pacing device. This kind of training was done also for session 3 and 4.

As it can be seen in Figure 1, from the first to the last session he was able to increase RSA as showed in respiration and HR oscillations occur almost in phase, even if his average respiration rate during feedback sessions decreased from 17 breaths per minute to 12-14 (so, not at the resonant frequency).

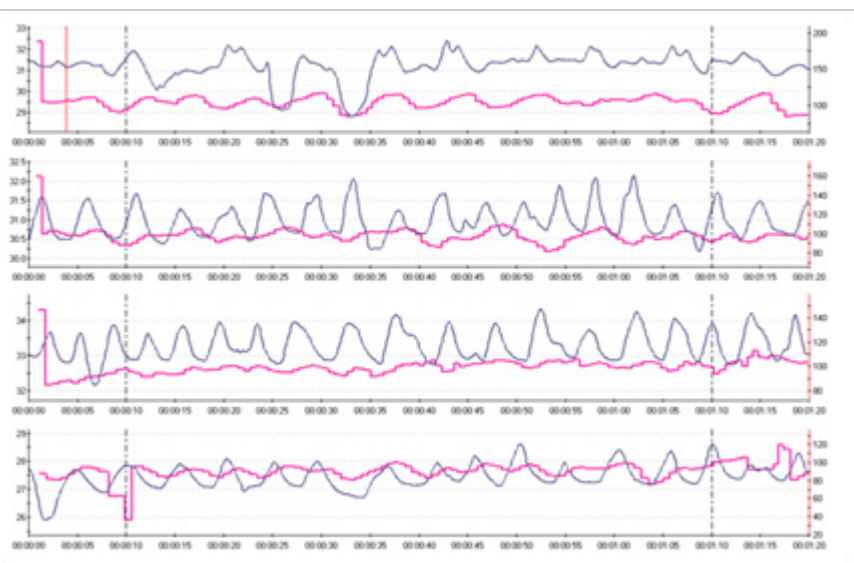

Figure I Post-training screenshot from session I to 4.The blue-line rapresents the abdominal breathing and the red line the heart rate tachogram.

The results showed that the SDNN were $23.64 \mathrm{~ms}$ and $38.46 \mathrm{~ms}$ respectively at baseline session 1 to session 4 and (Figure 2) the ratio between the power of LF and HF bands (vagal) activities is changed from 2.43 to 0.97 . This shows better overall balance between sympathetic and parasympathetic.

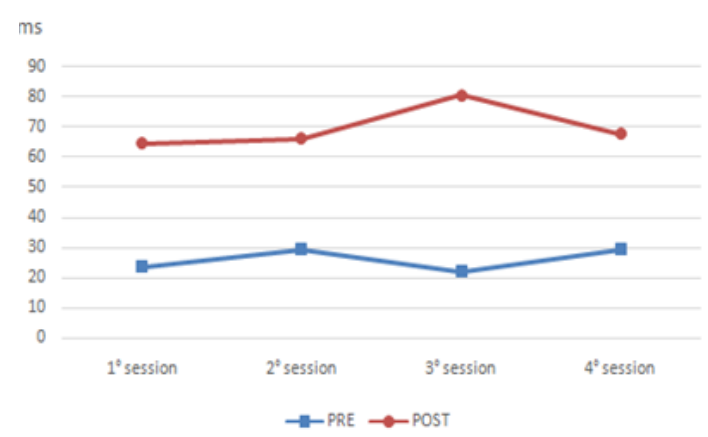

Figure 2 Pre and Post-training SDNN median millisecond (ms) levels from session I to 4 .
From session 3 to 4 surface electromyography (sEMG) training was also included to decrease neck muscle tension and promote relaxation. Before BF training, it was explained to Mario the key role of muscle tension in pain and he was trained in relaxation exercises focusing his attention to the feeling of relaxed muscles by classical Progressive Muscle Relaxation (PMR) methodology, delivered in a mini-format. The sEMG values (measured since the first session even if without specific focused training on this parameter) changed from $7.36 \mathrm{uV}$ to $3.20 \mathrm{uV}$, (from $3^{\text {rd }}$ session $5.16 \mathrm{uV}$ ) reflecting an increase in awareness about muscle tension and also stimulate blood flow of his body (Figure 3).

uv

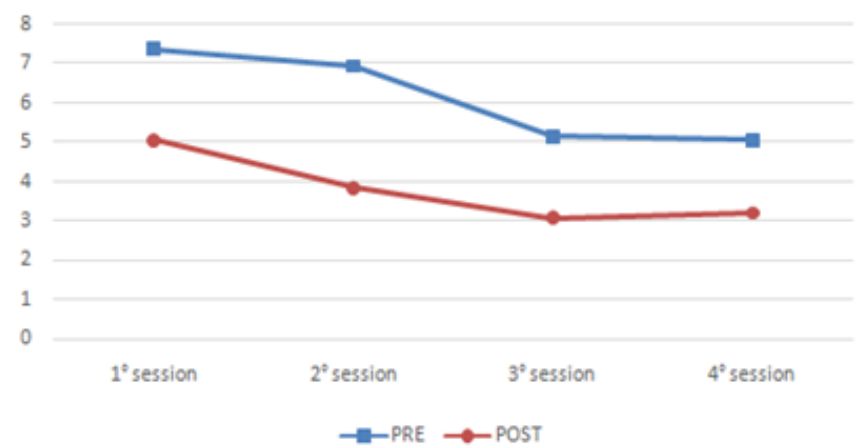

Figure 3 Pre and Post-training sEMG median microvolt (uV) levels from session I to 4 .

\section{Discussion}

The purpose of this case study was to evaluate a brief protocol for the identification of potential contributions for improving outcomes in cancer pain. In particular this case suggests that biofeedback training may improve emotional regulation thanks also to one's own ability to manage and adapt physiological arousal in response to situational demands. ${ }^{19,20}$ Significant differences observed in the sEMG and HRV measures could effectively reflect a decrease in negative psychological symptoms related to pain, thus to be able to reframe the personal pain perception.

Usally a main goal of biofeedback training is the generalization of skills, but given the short time planned to address this specific situation and from other similar experiences reported in the literature. ${ }^{13}$ Burton et al., ${ }^{21}$ even also with a single one BF session, ${ }^{10}$ we wanted to test this brief training in an exploratory manner.

As Benore et al. ${ }^{22}$ suggested, the knowledge gained from a biofeedback session can be used to reconceptualize their own anxiety and active monitoring through an increase in awareness, and not least, to allow parents to use other strategies (i.e. reminding to use breathing apps, doing preparatory exercises) to support their adolescent in pain.

Even though the situation itself (invasive procedure or treatments that cause pain) is not entirely under the personal's control, the adolescent's control of his breathing and his specific muscle-relaxation can be autonomously controlled. ${ }^{13}$

Psychologists and nursing staff should work closely to provide a better caring, and therefore simple, accessible and engaging tools are needed to serve both as a guide and as a coach to children undergoing anxiety-provoking and painful medical procedures: ${ }^{23}$ biofeedback training connected with relaxation, delivered in the way presented in this case repost, could be a good compromise. 


\section{Conclusion}

In conclusion, we want to highlight two aspects of the usefulness of the combined intervention of relaxation and biofeedback: a) as a system to increase individual awareness of the connection between emotions, physiological changes, and self-regulation; and b) as an adjuvant treatment, within an approach to integration multidisciplinary, interdisciplinary, and transdisciplinary - which can fit well in the DH's clinical practices.

\section{Funding details}

Alessandro Failo is supported by a Postdoctoral Research Fellowship from the University of Trento with the grant from Fondazione Trentina per la Ricerca sui Tumori (FTRT).

\section{Acknowledgements}

We thank Dr. Laura Collino and Dr. Chiara Rattin who have been and continue to be invaluable to this project.

\section{Conflict of interests}

Authors declare that there is no conflict of interest.

\section{References}

1. Campbell V. The challenges of cancer pain assessment and management Ulster Med J. 2011;80(2):104-106.

2. Oldenmenger WH, Sillevis Smitt PA, van Dooren S, et al. A systematic review on barriers hindering adequate cancer pain management and interventions to reduce them: a critical appraisal. Eur $J$ Cancer. 2009;45(8):1370-1380.

3. Chaturvedi SK, Peter Maguire G, Somashekar BS. Somatization in cancer. Int Rev Psychiatry. 2006;18(1):49-54.

4. Claar R, Walker L, Smith C. The influence of appraisals in understanding children's experiences with medical procedures. Journal Of Pediatric Psychology. 2002,27(7):553-563.

5. Chotolli M, Luize P. Non-pharmacological approaches to control pediatric cancer pain: nursing team view. Revista Dor. 2015;16(2):109113.

6. Lehrer P, Eddie D. Dynamic processes in regulation and some implications for biofeedback and bio behavioral interventions. Appl Psychophysiol Biofeedback. 2013;38(2):143-155.

7. Stubberud A, Varkey E, McCrory D, et al. Biofeedback as prophylaxis for pediatric migraine. A meta-analysis. Pediatrics. 2016;138(2):1-13.

8. Powers S, Mitchell M, Byars K, et al. A pilot study of one-session biofeedback training in pediatric headache. Neurology. 2001;56(1):133.

9. Stern M, Guiles R, Gevirtz R. HRV biofeedback for pediatric irritable bowel syndrome and functional abdominal pain: a clinical replication series. Appl Psychophysiol Biofeedback. 2014;39(3/4):287-291.
10. Myrvik M, Campbell A, Butcher J. Single-session biofeedback-assisted relaxation training in children with sickle cell disease. J Pediatr Hematol Oncol. 2012;34(5):340-343.

11. Achterberg J, Kenner C, Lawlis G. Severe burn injury: A comparison of relaxation, imagery and biofeedback for pain management. Journal Of Mental Imagery. 1988;12(1):71-87.

12. Knox M, Lentini J, McGrady A, et al. Game-based biofeedback for paediatric anxiety and depression. Mental Health In Family Medicine. 2011;8(3):195-203.

13. Shockey D, Menzies V, Glick D, et al. Pre procedural distress in children with cancer: an intervention using biofeedback and relaxation. J Pediatr Oncol Nurs. 2013;30(3):129-138.

14. Thompson M, Thompson L, Reid-Chung A. Chapter 8: Combining LORETA Z-Score Neuro feedback with Heart Rate Variability Training. In: $\mathrm{R}$ Thatcher, et al. editors. Z Score Neuro feedback. New York: Elsevier Inc; 2015. p. 159-188.

15. Yasuma F, Hayano J. Respiratory sinus arrhythmia: why does the heartbeat synchronize with respiratory Rhythm? Chest. 2004;125:683690.

16. Steffen RS, Austin T, DeBarros A, et al. The impact of resonance frequency breathing on measures of heart rate variability, blood pressure, and mood. Front Public Health. 2017;5:2296-2565.

17. Koenig J, Loerbroks A, Jarczok M, et al. Chronic pain and heart rate variability in a cross-sectional occupational sample: Evidence for impaired vagal control. Clin J Pain. 2016;32(3):218-225

18. Alfvén G, Grillner S, Andersson E. Children with chronic stress-induced recurrent muscle pain have enhanced startle reaction. Eur J Pain. 2017;21(9):1561-1570.

19. Appelhans BM, Luecken LJ. Heart rate variability as an index of regulated emotional responding. Review of General Psychology. 2006;10(3):229-240.

20. Munafò M, Patron E, Palomba D. Improving managers' psychophysical well-being: effectiveness of respiratory sinus arrhythmia biofeedback. Appl Psychophysiol Biofeedback. 2016;41(2):129-139.

21. Burton K, Morrow A, Beswick B, et al. The feasibility of using the brighthearts biofeedback-assisted relaxation application for the management of pediatric procedural pain: a pilot study. Pain Practice. 2018.

22. Benore $\mathrm{E}$, Banez G, Sawchuk T, et al. Applied biofeedback in pediatric pain. Biofeedback. 2014;42(3):96-102.

23. Cheryl HT Chow, Ryan J Van Lieshout, Louis A Schmidt, et al. Systematic Review: Audiovisual Interventions for Reducing Preoperative Anxiety in Children Undergoing Elective Surgery. J Pediatr Psychol. 2016,41(2):182-203 